\title{
A Student-facing Dashboard for Supporting Sensemaking about the Brainstorm Process at a Multi-Surface Space
}

\author{
Andrew John Clayphan \\ The University of Sydney, \\ NSW, 2006, Australia \\ Andrew.Clayphan@sydney.edu.au
}

\author{
Roberto Martinez-Maldonado \\ University of Technology Sydney, \\ NSW, 2007, Australia \\ Roberto.Martinez-Maldonado@uts.edu.au
}

\author{
Judy Kay \\ The University of Sydney, \\ NSW, 2006, Australia \\ Judy.Kay@sydney.edu.au
}

\begin{abstract}
We developed a student-facing dashboard tuned to support posthoc sensemaking in terms of participation and group effects in the context of collocated brainstorming. Grounding on foundations of small-group collaboration, open learner modelling and brainstorming at large interactive displays, we designed a set of models from behavioural data that can be visually presented to students. We validated the effectiveness of our dashboard in provoking group reflection by addressing two questions: (1) What do group members gain from studying measures of egalitarian contribution? and (2) What do group members gain from modelling how they sparked ideas off each other? We report on outcomes from a study with higher education students performing brainstorming. We present evidence from i) descriptive quantitative usage patterns; and ii) qualitative experiential descriptions reported by the students. We conclude the paper with a discussion that can be useful for the community in the design of collective reflection systems.
\end{abstract}

\section{CCS CONCEPTS}

- Human-centered computing $\rightarrow$ User studies; Laboratory experiments; Computer Supported Cooperative Work

\section{KEYWORDS}

Reflection, collaboration, brainstorming, dashboard, sensemaking. ACM Reference format:

A. Clayphan, R. Martinez-Maldonado, and J. Kay. 2017. A Studentfacing Dashboard for Supporting Sensemaking about the Brainstorming Process at a Multi-Surface Space. In Proceedings of the $29^{\text {th }}$ Australian Conference on Human-Computer Interaction, Brisbane, QLD, Australia, November 2017 (OzCHI 2017), 10 pages. https://doi.org/10.1145/3152771.3152777

\section{INTRODUCTION}

In group work, people leave large amounts of digital traces behind whilst interacting with their digital devices. It has been

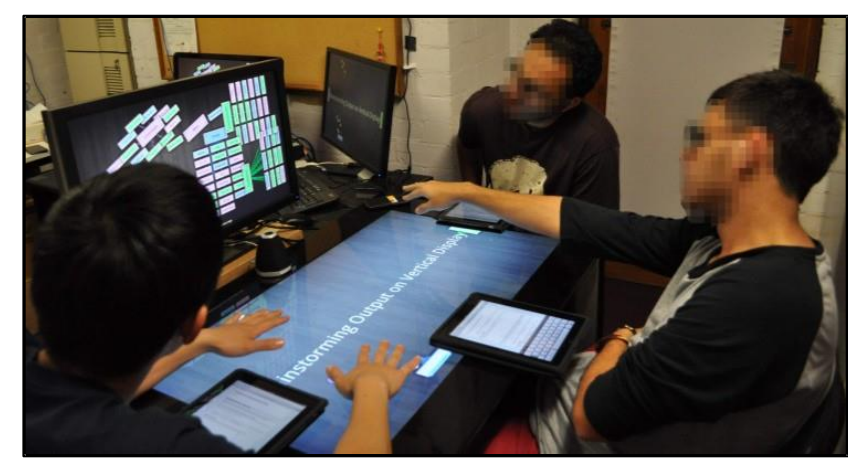

Figure 1: A team reflecting on their brainstorm experience as they view the output of their session on the studentfacing dashboard (shown left of center).

demonstrated that analysing alternative views of these captured activity data can provide effective support for reflection when shown to either participants [10,33] or facilitators [35]. This is particularly important for building meta-cognitive skills to enable people to reflect on their contributions as a group (and individually), and can lead to an increase in visibility, awareness and accountability [24]. A wide range of techniques for modelling learning processes has long been used to support reflection on the basis of developing knowledge, skills and performance [9]. Our approach draws on key literature about Cooperative and Competitive visualisations [26, 47, 49] and on Open Learner Models (OLMs) [11, 12, 17], with the aim to enable groups to become aware of key activity aspects. Reflection involves actively monitoring, evaluating and modifying peoples' understanding of processes. Schön [48] highlights the value of reflection-on-action, which involves thinking back on what has been done in order to discover how various actions have contributed to actual outcomes.

Multi-touch tabletops (e.g. Fig. 1) have proven effective in facilitating face-to-face group brainstorming for small-groups and can be used to support the free flow of ideas $[6,14,47]$. These shared interfaces enable people to generate ideas in parallel, interact with digital representations of these and save their work for future revision. Interactive tabletops provide new ways to tackle group problems such as free riding, social loafing and idea blocking [47]. This includes coding outputs to show contributions, and the use of multiple inputs to mitigate the need for turn taking $[15,27]$. A less explored potential is combining interactive tabletops with large vertical displays to present key visual indicators based on the modelled processes of the collaborative activity to support group reflection. This is important as different display formats offer varying advantages, functionalities and limitations in support of group work [18, 25]. 
This paper describes the design and validation of a studentfacing dashboard that contains visual representations of models of the group of learners' interactions (Open Learner Models OLMs) to support groups in giving meaning to their own collaborative experience. By definition, OLMs make a computer representation of the learner's data available as an important means of supporting learning [11]. The design of our dashboard is aimed at helping group members who had just completed a tabletop-based brainstorming session to reflect on their work, to appreciate the processes that operated within the group, and their contributions and roles. We report on outcomes from a study with higher education students performing brainstorming at a multitouch hybrid system that consists of a vertical screen and a horizontal interactive tabletop. We present evidence from two sources: i) descriptive quantitative usage patterns; and ii) more interpretive qualitative experiential descriptions reported by students. The study illustrates the usefulness of showing multiple, different perspectives about the data models that show evidence of how the group members engaged in collaborative face to face ideation tasks.

This work is guided by two core research questions drawn from brainstorming theory $[4,22,44]$ (that we motivate in the following sections): $R Q 1$ ) What do group members gain from studying egalitarian contribution visualisations? and $R Q 2$ ) What do group members gain from modelling how they sparked ideas off each other? The first aims to find out whether people who have considered the final outcome of the brainstorm session modify their initial self-assessment and their understanding of this, after viewing different visual representations of traces of their contributions. The second question aims to assess if a scaffolding process using the dashboard (that provides OLMs and prompts reflection tasks) can help group members gain understanding about how they built on each other's ideas compared with observing just the final product outputs. Here, each individual group member works to consider the effects of being in a group.

The rest of the paper is organised as follows. The next section describes related work in terms of technological support for reflection in groups and the work on OLMs underpinning our study. Section 3 describes the design of our toolset for supporting both brainstorming and reflection-on-action. Section 4 describes the study with higher education students performing creativity tasks. Section 5 presents a discussion of the results of the study. Section 6 concludes the paper with a summary and ideas for future directions following this work.

\section{RELATED WORK}

\subsection{Multi-touch Tables and Reflection Tools}

Tabletops are large-horizontal surfaces that allow parallel interaction in a face-to-face setting. Previous research has explored how tabletops can be suitable platforms for reflection. Kharrufa et al. [29] showed that tabletops can support postactivity reflection, such as group process replay, and bookmarks to critical group moments. Both allow chances to increase student awareness and provide opportunities for rich discussion. They demanded modest additional time obligations when used as part of a regular classroom activity. Do-Lenh et al. [21] explored how educational tabletop systems affect reflection, how reflection can be orchestrated within classrooms, and the impacts on learning as well as effectiveness. For example, the use of a wall display with a simple visual, to indicate peer progress within the classroom. Al-Qaraghuli et al. [1] showed how visualisations can be used to detail actions over time to foster analysis of followed processes. Martinez et al. [36] showed how a set of models shown to teachers, could be used to identify different levels of collaboration and in a series of follow-up studies [34, 37] presented the use of a portable dashboard with real-time data on collaboration aspects as part of a classroom infrastructure, finding that simpler models are often preferred within more complex environments. Tausch et al. [49] explored co-operative and competitive visualisations displayed during a brainstorm, investigating effects on collaboration, and Clayphan et al. [17] explored paper-prototyped visualisations which drew on activity logs combined with audio speech to help people reflect on key aspects of the brainstorm process.

The work presented in this paper is the first to date, that we are aware of, to utilise a tabletop and a display to promote brainstorming reflection, to measure group effects by calling on people's assessment of the process.

\subsection{Open Learning Modelling in Dashboards}

To enable learners to have access to representations of their knowledge, OLMs provide a means for promoting benefits such as reflection and metacognition [12]. Allowing individuals to contribute to their own or their peer's model can promote reflection by confronting one another with their understanding about a problem. OLMs can promote discussion and facilitate collaborative interaction [10].

Providing multiple OLM representations can invite learners to reflect on their performance in different ways [33]. This can support higher levels of reflection, as different learners may naturally prefer different OLM forms. In particular, if learners are aware of the benefits of the OLMs and how they relate to an activity or assessment, benefits of reflection and learning, individually or as a group may be achieved [7, 9]. OLMs themselves do not need to be complex to have benefit; simple scrutable models can provide feedback on learning products and processes, both of which promote reflection [32]. One such example is the exploration of how an intelligent tutoring system can help learners in a brainstorm, through the use of heuristicbased feedback; and community data-driven social recommendations [50].

Although there has been renewed interest in exploring how OLMs can support metacognition [2, 12] visually and in combination with other learning analytics innovations $[8,28]$ there has not been substantial empirical work demonstrating possible uses of OLMs for supporting group activity [13]. Through our study, we explore how OLMs can be used to provide aid to students to reflect on critical collaborative brainstorming processes. 


\section{DESIGN}

The design for our student-facing reflection dashboard draws on group brainstorming research [5, 42]. Group brainstorming is a creative problem technique widely used for coming up with solutions to diverse problems [40]. Brainstorming primarily consists of two distinct phases. The first is a divergent idea generation phase, where members formulate and share as many ideas as possible. This is followed by a convergent idea categorisation-refinement phase, where the ideas are assessed, organised and filtered.

The technique aims to help produce many innovative ideas and make sense of them. It relies on a small set of core rules: (1) no criticism; (2) unusual and wild ideas welcome; (3) quantity is encouraged; and (4) combination and improvement of ideas is sought. When these rules are followed, research [43, 45] has shown that group members are able to contribute in a criticismfree environment, often leading to greater participation and more diverse outputs [51]. Furthermore, there is evidence that suggests the ideas generated by each member activate related idea associations in other members, a process referred to in the literature as idea sparking [4, 23, 31, 39].

We are particularly interested in exploring the potential of idea-generating groups in two key areas of brainstorming: 1) task performance measured as idea contributions [45] (see $R Q 1$ ) and 2) the effect of idea sharing, exploring whether members stimulate each other's creativity [31] (see $R Q 2$ ). To do this, we extended our existing tabletop brainstorming system $[15,18]$, which has been run successfully in-the-lab and in-the-wild, by developing a student-facing dashboard to scaffold sensemaking as a six-step reflection activity to happen right after the group activity. In the following subsections we describe how the design of the six steps of our reflection dashboard are directly linked to our core research questions.

\subsection{Reflection on Egalitarian Contributions (RQ1)}

We choose three OLM representations to be included in our dashboard, looking at what could be made available to a group upon finishing their brainstorm. We choose the final output and two temporal items. In the reflection, we present each item in turn, with students asked the following: 'I contributed an equal amount to the brainstorm?' Students reported their answers on a Likert scale (1 to 6), with space for explaining self-assessment choices. These are described in Steps 1-3 of the reflection.

Step 1) The 'final brainstorm activity output' is shown to the group on the vertical display (Fig. 2). This represents the end snapshot from the tabletop. The orientation and colours of the ideas are unchanged. This is what a group sees at the conclusion of their activity, the interface supporting some form of awareness around group contribution from the visual elements present. This is included as it helps to expose underlying beliefs and premises of one's self-assessment [19, 38] of the posed question. This provides an opportunity to later explore if self-assessments change with other visual forms made available.

Step 2) A 'contributions chart' is shown to the group in the form of a pie chart (Fig. 3). This was chosen based on

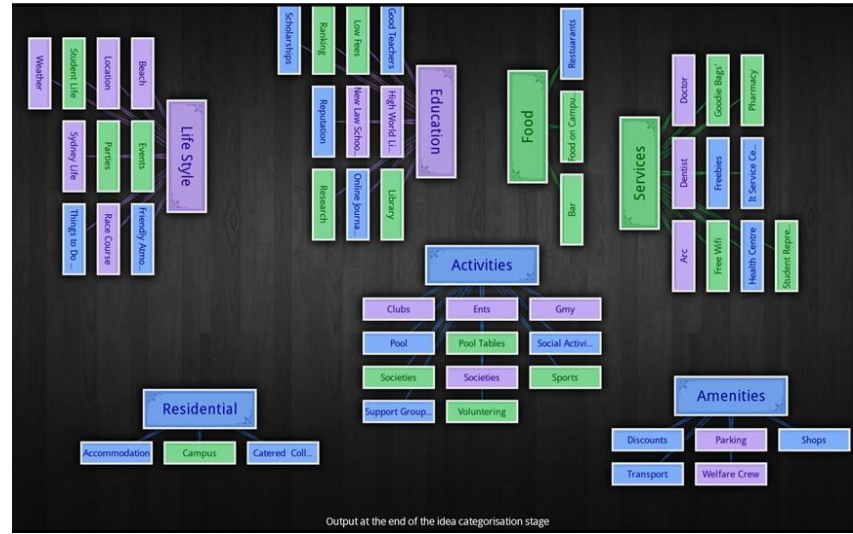

Figure 2: Brainstorm Outputs (Step 1 and 4).

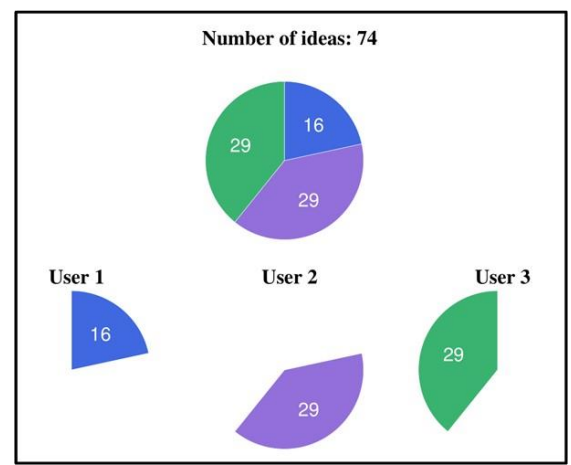

Figure 3: Contributions Chart (Step 2).

representation familiarity, interpretability and ease of use. This visual is used to show proportionality of contributions, in terms of the ideas created, is colour coded to the same colour as the idea widgets from each individual, and provides the count of ideas for each person, so as to not be afflicted by interpretation issues [30]. This representation is meant to push people to think in new ways, develop explanations around their experiences, and question why their output is, as it is.

Step 3) A 'contributions timeline' is shown to the group (Fig. 4). This shows contributions grouped into half-minute intervals, with lines are colour coded to show each individual. This representation is presented to aid each person in understanding

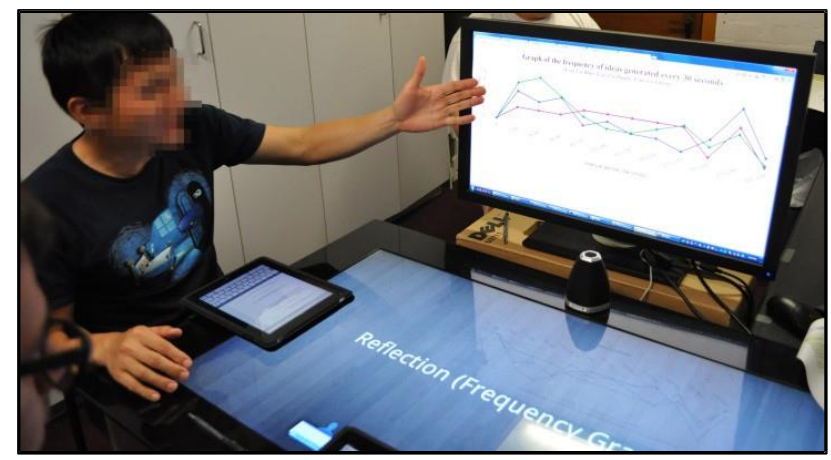

Figure 4: Contributions timeline (Step 3). 
their relative contributions and when they contributed, to help promote an understanding of their behaviour and outputs throughout their ideation [3].

\subsection{Reflection on Group Effects (RQ2)}

For the second theme, we also choose three items to present. The first was a final output summary, the second a video replay of the activity, and the third, an explicit classification activity, drawing on the judgements of each of the group members to inform the system of which ideas were sparked, with supporting visuals. In the reflection, each item was presented in turn, with the following question asked 'Did you spark ideas off other people?' (where sparking was explained as ideas created due to the inspiration of someone else's idea - either due to hearing it or reading it from the pool of ideas created). This was answered on a Likert scale with space provided for explaining selfassessment choices. These are described in Steps 4-6.

Step 4) The 'final brainstorm activity output' is shown once again. As per Step 1, this is included as an initial visual, and provides the opportunity to later explore if self-assessments change when other OLM representations are made available.

Step 5) A 'brainstorm replay' (sped up by a factor of 4) is presented. This provided the unique opportunity to observe how the set of ideas and groupings evolved over time, seeing the ideation phase unfold, and the categorisations taking place. The replay speed was empirically chosen, balancing a trade-off of the time needed to review the process. This aspect was inspired by work on memory [46] and group visual feedback tools [20].

Step 6) A 'brainstorm classification exercise' is asked of the group, where group members nominate which of their ideas are sparked (that is, ideas spurred from the inspiration of others). A custom selector tool is provided on the tabletop (Fig. 5) for each member. The selector tool was purposely built to aid the classification process, and displays each individuals ideas in a time ordered sequence, with the ideas of other individuals around it. To further aid the exercise, the vertical display shows a topdown, left to right list of ideas ordered by time (Fig. 6). After this classification, a visual is shown on the vertical display regarding this aspect (Fig. 7). This gives an indication of the relative amount of inspiration drawn from group members. This exercise was inspired on literature looking at the effects of group collaboration

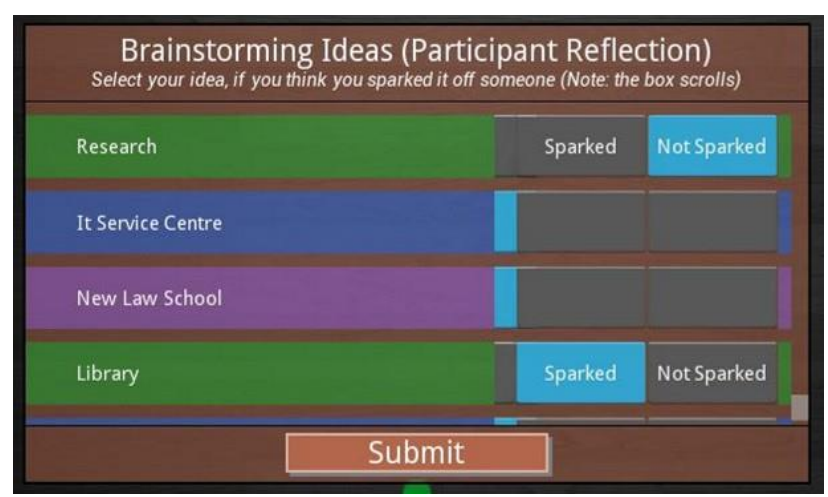

Figure 5: Classification Dialog (Step 6).
Brainstorming Output (time order: left to right)

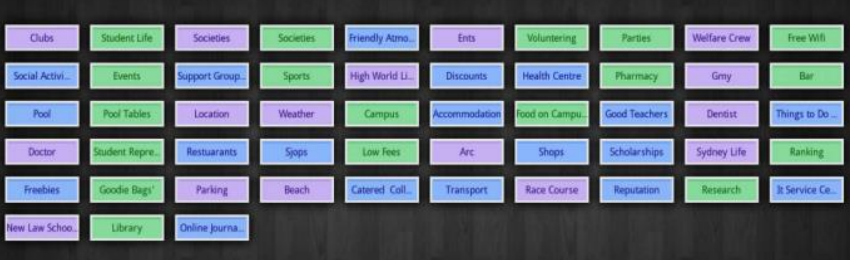

Figure 6: Top-down display to help classification (Step 6).

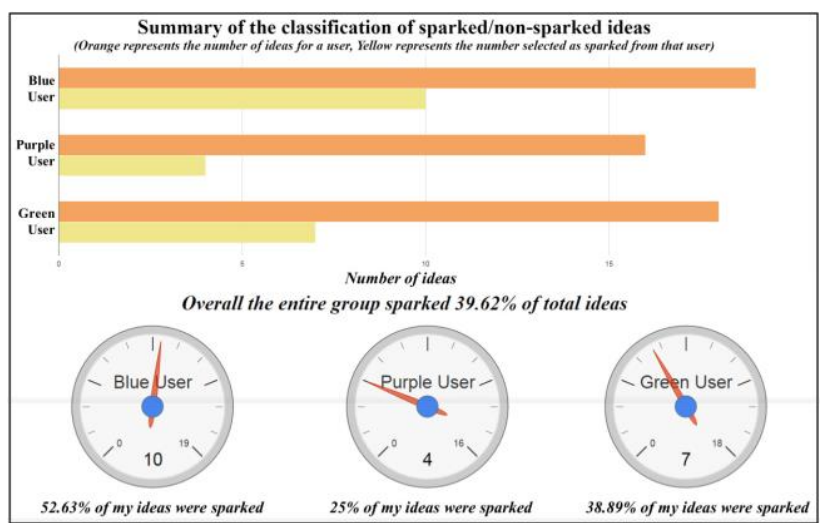

Figure 7: Summary from a classification task (Step 6).

on brainstorming $[39,45]$. To date, no other brainstorming system has asked members to nominate which ideas have been inspired by others, instead relying on external assessment or automatic methods.

\section{STUDY}

An in-the-lab user study linked to the lines of inquiry drawn from brainstorming literature was conducted to investigate the effects of different visual forms as part of a reflection process.

\subsection{Participants}

Thirty student volunteers were recruited from a local university and took part in the experiment in groups of three (mean age 22, age range: $20-27$ years, 23 males, 7 females). They were from varied degree majors including social studies, computer science, engineering and medicine. All volunteers reported familiarity with personal touch interfaces, such as smartphones and tablets. Students sat comfortably around the hardware setup (table and display) with equal viewing access to both (e.g. Fig. 1).

\subsection{Method and Procedure}

The study took place in a quiet room equipped with a 42 inch interactive tabletop (SUR40); a 27-inch vertical display; and separate wireless keyboards for each person (to input ideas to the brainstorming system). For the reflection activity, students sat around the table with the vertical dashboard in the center, such that each person could see it without obstruction. Each session was video recorded, with one camera positioned on the ceiling 
above the setup, and one camera to the side of the setup. Further, all typed input via the wireless keyboards was logged by the brainstorming application to a database for later analysis.

The rules of the brainstorming method were explained to students (see Section 3), with groups explicitly told to verbalise (announce) ideas as they entered them into the tabletop via their keyboards (note, the dashboard during this time did not display any information). The brainstorm had a time constrained ideation phase of 5 minutes and an untimed categorisation phase. The topic was: 'what do you think is important to high school students in motivating them to come to university'. After the brainstorm activity, the scaffolded reflection activities took place, where six items were shown, three items for 'reflection on egalitarian contributions' and three items for 'reflection on group effects'. After each visual, a question was asked in response to the reflection step on a Likert style scale from 1 to 6 . This was answered on supplied iPads (to facilitate data collection), with an accompanying question asking how the visual influenced their self-assessment. Following, a post-experiment questionnaire was issued (to each student separately), with questions about usability and for commentary about aspects they found most/least useful.

\section{RESULTS AND DISCUSSION}

In this section, we report on students' perceptions in relation to each step in the reflection activity, investigating changes in self-assessment ratings, time spent in each step, data from the post-experiment questionnaire, and commentary left. We note, no gender effects were observed with the given team formations.

\subsection{Post-Experiment Questionnaire}

The post-experiment questionnaire responses are presented in Fig. 8. Overall, there was a high level of understanding of all the aspects of the brainstorm and the reflection activity facilitated

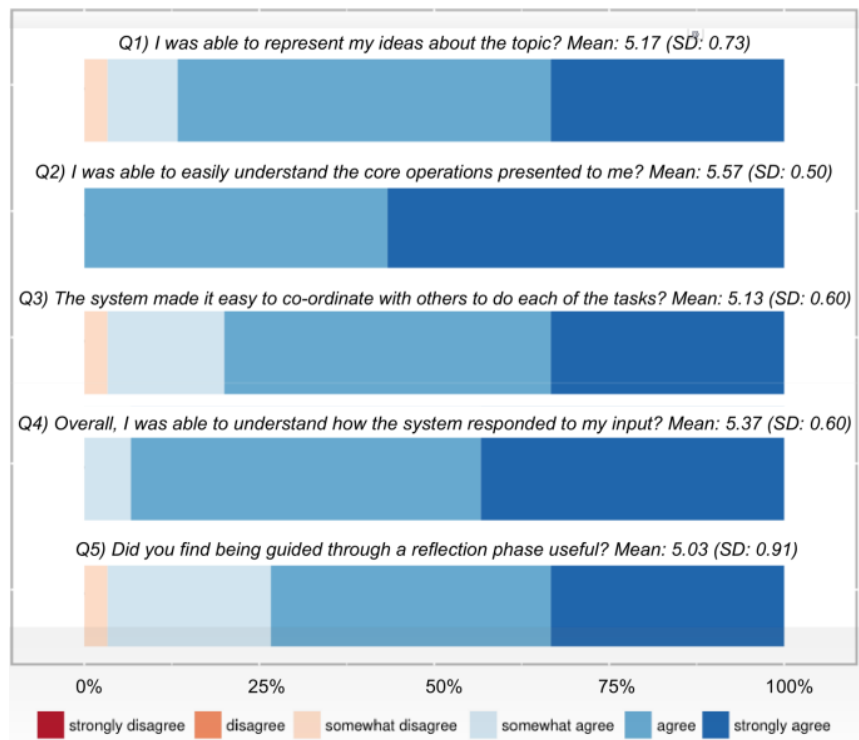

Figure 8: Results of the post-experiment questionnaire. by the student-facing dashboard (Questions $1-4$ ), with a mean rating of 'agree' On asking if students liked being guided through reflection, high satisfaction was recorded (Question 5 - 5.03/6), with free-form responses indicating 26/30 students had a positive experience with this aspect. Overall, the 'contributions chart' (Step 2) for the first reflection area, and the 'brainstorm classification exercise' (Step 6) for the second reflection area were most liked, as drawn from commentary nominated in the postquestionnaire. No comments indicated that there was difficulty in using the brainstorm system or the six-step reflection activity that followed, although 4 students (13.33\%) remarked on 'touch sensitivity' at the table.

\subsection{Time Spent on Reflection}

The mean time taken to complete the entire reflection activities took approximately 16 minutes (SD: 4.5 mins) (see Fig. 9). Overall, Steps 1-4 took approximately 2 minutes each, and Steps 5 and 6 took 4 minutes each. Of the ten groups, nine groups spent more time on reflection than the actual brainstorming task (which itself took a mean time of 11 minutes (SD: 1.5 mins)). On average, each group spent about 25 minutes together.

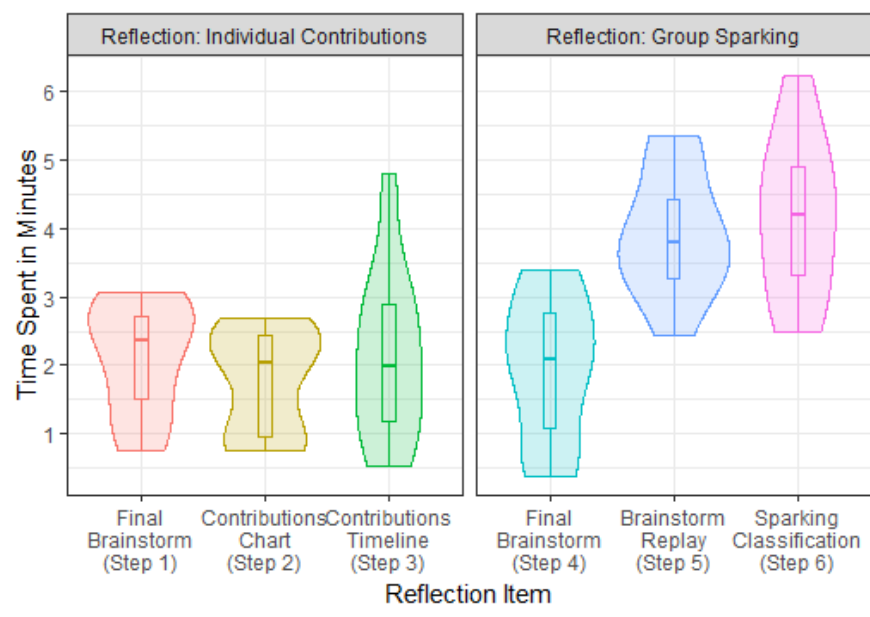

Figure 9: Time spent by students on each reflection step.

\subsection{RQ1 - What do people gain from studying egalitarian contribution visualisations?}

Table 1 reports on selected qualitative feedback for each reflective aspect (Steps 1-3) with Fig. 10 reporting the results of the self-assessment ratings. From the number of ideas generated, certain group strategies emerged: near equal ideas generated per person (Groups 2, 3, 5, 7 and 8); a dominant person (Groups 6 and 9) - which is where many ideas are created by one member than the other two in the group; and a straggler (Groups 1, 4 and 10), where one person has considerably less ideas in the group. These strategies were evident in the initial user self-assessment where the final brainstorm output was shown to the group.

In Step 1, students assessed their contributions, and this helped form the basis for comparisons of the later presented 
Table 1: Examples of externalised reflective statements on aspects of Egalitarian Contributions by selected students.

\begin{tabular}{l}
\hline Reflection on Egalitarian Contributions \\
\hline Step 1- Final Brainstorm Output \\
\hline 4A Based on the colour-coding, appears to be a fairly even \\
contribution spread at first glance. \\
8C I looked at the colours of the words and I think I came up with a \\
little more than a third of them. \\
9A There isn't a lot of blue but there is a lot of the other colours. \\
\hline Step 2 - Contributions Chart \\
\hline 1A Did worse than I thought. \\
5A The results showed that I contributed the same amount \\
compared to the other participants. The average number of \\
ideas were around 26-27 ideas and I was in that average. \\
\hline Step 3-Contributions Timeline \\
\hline 4A This chart, showed the peaks and troughs on my contribution \\
over time - it made it clear to me, that I wasn't consistent (I \\
thought I was). \\
5B I was very consistent throughout the brainstorming process. I \\
feel like my contributions were appreciated. \\
7A This graph made me feel like I contributed more than the \\
information presented in part 1 [the brainstorm output], but it \\
made me also feel like I did less than what I saw in part 2 [the \\
pie chart]. Sometimes, my contribution frequency was close to \\
zero, but other times, it was really high. This graph made me \\
realise my contributions were done in bursts.
\end{tabular}

visuals. The bulk of the ratings were near the middle, with those rating themselves higher or lower corresponding to the dominant and straggler members. In the self-assessment, 22/30 (73.33\%) contributed a comment indicating the final brainstorm output form aided them in understanding their contribution to the group. Students primarily commented on colour distribution and equality of different members ideas within categories (e.g. Table 1'Step 1'), with comments revealing 'gut intuition' and 'memory' as bases for self-assessment. This suggests that only showing the final brainstorm output alone to group members may provide very limited evidence for promoting critical reflective thinking, as such, other forms of evidence are needed.
Next, students were shown the contributions chart (Step 2) with the segments indicating each person's contribution. For this, 23 out of 30 students $(76.67 \%)$ reported insight, measured by either a change in their self-assessment score or from explicit written feedback. We can see the distribution shifts (e.g. Fig. 10), with written responses (e.g. Table 1-'Step 2') revealing knowledge discovery and back-talk [48]. For example, with comments commencing with the phrases: "it was less than I thought...", "I just found out..."; "I just realised...". This illustrates awareness. For nine students, even though selfassessment ratings did not change, they left a comment regarding the utility of the chart. For these, it served to re-affirm their understanding and their initial belief of their contribution to the group.

Lastly, students were shown a contributions timeline (Step 3). Seventeen students (56.67\%) noted an effect, judged by a selfassessment Likert score change and from contributed feedback. Ten students altered their self-assessment score (relative to their rating nominated in the previous step). Comments (e.g. Table 1'Step 3') indicated that this visualisation allowed students to see their developing proficiency in the group brainstorm and better understand the approaches they took when creating ideas. Interestingly, after seeing this visual, the resultant rating distribution was very similar to that of Step 1 .

In summary, students gained awareness of their performance from the reflection phase which was not possible from just viewing the final state of the brainstorm output. This points to the value of multiple visualisations as a way to promote key reflective processes. The contributions chart helped students reflect about real contributions to the group brainstorm, with many updating their self-assessments as a result. The contributions timeline helped students reflect on the process, and triggered rich discussions between group members. In short, this suggests that presenting summarised information to group members in terms of modelled individual participation relative to the group provides them with more evidence to make more accurate judgements about their performance within their group.

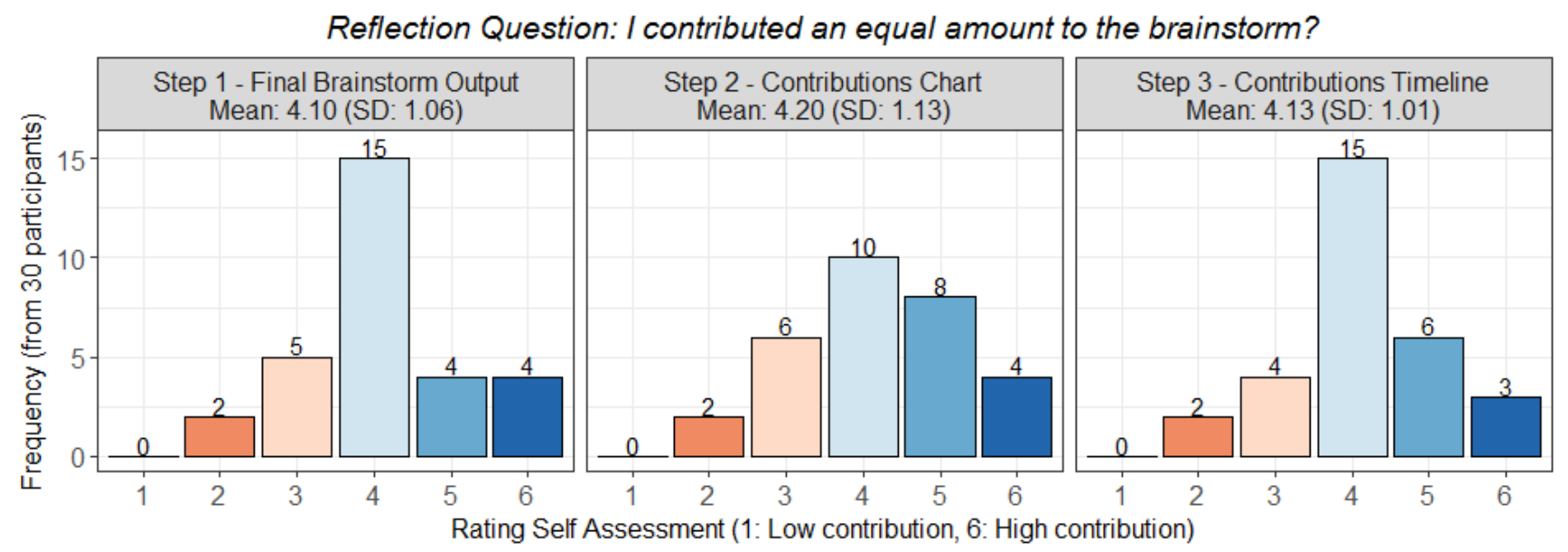

Figure 10: Student self-assessment ratings as a part of their reflection on Egalitarian Contributions. 
Table 2: Examples of externalised reflective statements on Group Effects (Idea Sparking) aspects by selected students.

\section{Reflection on Group Effects (Idea Sparking)}

Step 4-Final Brainstorm Output

4A From memory and observation of the display, I think about half my ideas were sparked.

7B Felt like I sparked a lot of ideas off others and judging from the way we cateorised the different ideas as shown, I would say that's the case.

8B Overall collaboration during the brainstorming exercise increased my frequency of ideas. Listening to an idea/word someone spoke I was able to be more creative and complement it [their idea] with others.

\section{Step 5 - Brainstorm Replay}

2A Helps my memory.

$2 B$ Lets me see how we did the brainstorming.

$4 \mathrm{~A}$ This helped remind me of the mental processes I went through in the braisntorm and reinforced that a number of ideas were sparked.

5B I found that I did take some ideas from others.

7A Made me feel like I sparked more ideas off others, mainly because it helped me remember some ideas were actually sparked off others, when I originally thought I had come up with them on my own.

Step 6 - Classification Exercise (and Visual)

$2 \mathrm{~A}$ Interesting to see I sparked a quarter of my ideas.

7B This exercise confirmed my feeling about my sparked ideas, especially after just seeing the replay.

9C There were actually even more ideas been sparked off [others], when I looked at the ideas in detail.

\subsection{RQ2 - What do people gain from modelling how they sparked ideas off each other?}

The second question explores the impact of scaffolding reflection by helping group members' recall how their ideas evolved and how group members sparked ideas off each other. Table 2 reports selected comments from this aspect of the reflection (Steps 4-6) with Fig. 11 reporting the results of the selfassessment ratings. Sparked ideas (as classified) from individuals range $\mathrm{d}$ from $0 \%-69 \%$ (Mean=38\%, $\mathrm{SD}=15)$, where $0 \%$ indicates a person thought none of their ideas were sparked or inspired from group members. The total number of ideas sparked per group ranged from $22 \%-47 \%$ (Mean $=35 \%, \mathrm{SD}=8$ ). For each brainstorm, the mean number of ideas created in a group was $47.4(\mathrm{SD}=17)$. Given the explained rules of the method, it is not too surprising, a third of the ideas on average were elected as sparked within each group.

For reflection on group effects, students were shown the final output of their brainstorm (Step 4). Roughly more than half nominated in their self-assessment, that they drew inspiration from other group members. Combined with comments left (e.g. Table 2- 'Step 4'), in total 23 twenty-three students (76.67\%) indicated they felt they had sparked ideas, with assessments pointing to internal intuition. For example, a couple of students stated this as follows: "I felt like I sparked a lot of ideas off others" and "listening to an idea allowed me be more creative".

Following this visual, a replay of the brainstorm was provided (Step 5). Twenty students (66.67\%) stated that the replay triggered some form of insight. Comments by two students, exemplify this: "it remind[ed] me of [my] mental processes [and] reinforced that a number of [my] ideas were sparked [from those by others]"; and "it helped me remember ideas that were actually sparked, that I thought were original". The value of the replay was also reflected in the change in the distribution of ratings (e.g. Fig. 11) with students revising their ratings to either end of the spectrum. Interestingly, the replay was commented by three students, having a lengthy duration relative to other reflection aspects.

Regarding the last aspect (Step 6), students went through their ideas, indicating if these were inspired by others' ideas. This allowed students to deeply engage with understanding the process of sparking. For example, when students viewed the final brainstorm output, two of them commented that 'sparking likely only occurred at or towards the end of a brainstorm'. However after the classification exercise, it became apparent to those same students that sparking was distributed throughout the brainstorm activity. Similar to the replay, the time required to do the

\section{Reflection Question: Did you spark ideas off other people?}

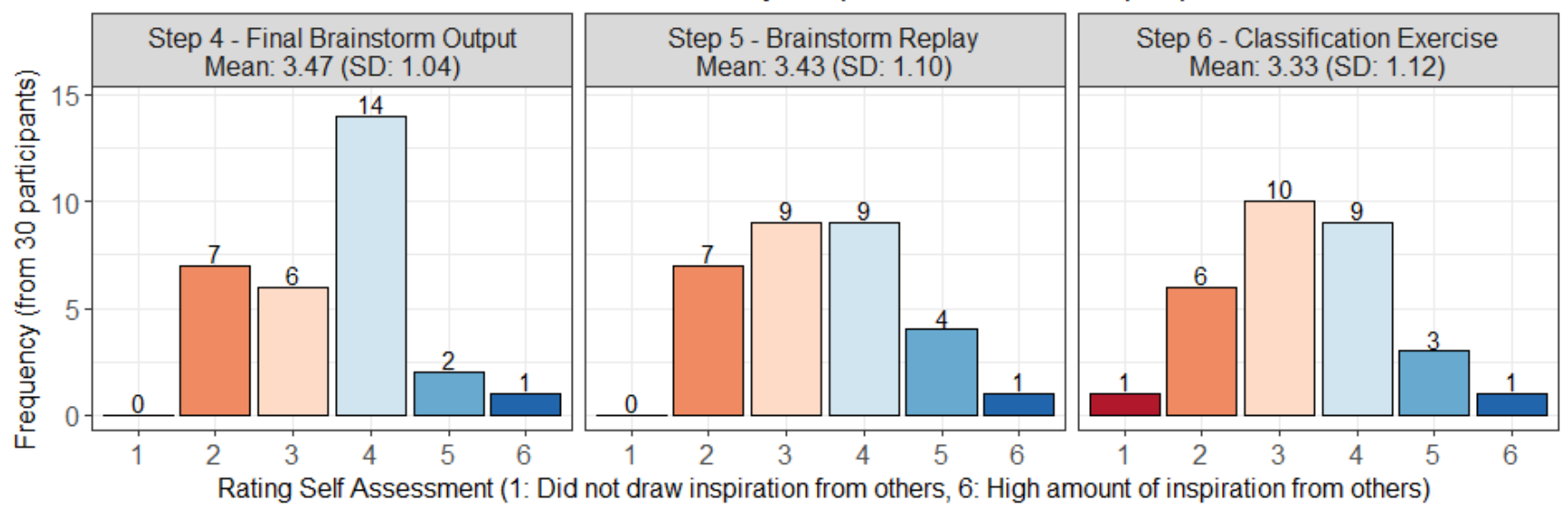

Figure 11: Student self-assessment ratings as a part of their reflection on Group Effects. 
classification, had a mean time of 4 minutes, which gave rise to a small number of comments, such as "it was long and laborious", but students did also acknowledge its benefit, for example, with comments like the following "[while it] was quite long, it was useful to think about the sparked ideas". This is a trade-off, with certain aspects of reflection needing more time than others. If a facilitator (or teacher), wants a group to reflect on this aspect, this time may be justified. We note the distribution of ratings did not heavily change. It appears in Step 5 and 6, these forms served to normalise initial assessments.

From the qualitative feedback collected, both the replay (Step 5) and the classification exercise (Step 6) were used in different ways. The brainstorm replay allowed students to revisit processes undertaken throughout the entire brainstorm. The classification exercise helped students deeply consider which of their ideas were due to being part of a group. This helped uncover misconceptions - such as the extent to which the group sparked ideas from one another and when. The results of both representations confirm the difficulty in attempting to measure sparking (which prior works have theorised and tried to do automatically $[16,41])$, and indicate the benefits of such an exercise as part of a dedicated reflection period.

\subsection{Limitations}

Our experimental design involved some trade-offs. Notably, our study was conducted in a lab setting, which is different to an in-the-wild setup. For example, there were no hard time limits on each of the six reflection steps (this was on purpose), nor were their explicit learning objectives (which would likely be the case if the tool was deployed to a classroom). For the topic we choose "what do you think is important to high school students in motivating them to come to university', this was relevant to the set of volunteers whom took part in the study (all being students of the university). The experiment in its current form, thus allowed us to observe how long a group spent when exploring different visual representations - a finding most useful for the adoption of such interfaces in the future, particularly in classrooms or other domains where time is an issue. We also note that while insights were found in these single session reflection runs, it would be interesting to track insights surrounding the behaviour of groups over the long term.

We also acknowledge that the dashboard presented in this paper is one among many forms of dashboards, and visual representations that we could have chosen. While we considered other visuals, this work built on our previous work [16] which paper-prototyped a reflection interface. It was found in the earlier study that simple visuals worked best, relative to complex models of how to display and communicate information, and this guided part of the design for this system. In addition, this study moved past the paper prototype as the next step to understanding use as part of real collaborative group system (in this case a brainstorm scenario). We wanted to make use of the table's potential affordances for users to sit face-to-face, to help with prompting discussion during reflection, augmented with that of a center facing vertical dashboard. Though at the same time, as acknowledge that the system may be limited in terms of the numbers of people it can support, these are future research lines of inquiry that may be interesting to explore.

\subsection{Summary}

When group members were shown the outputs from the brainstorm activity, immediately they formed an internal mental model of how much they believed they contributed, and the effect of working and being part of a group. To gauge initial beliefs, students used features present from the brainstorm interface, such as the colour of the individual ideas created. However, with the introduction of even simple visual models examining two key aspects of collaboration from the group activity (participation and sparking effects), groups drew extra insights over their processes. The egalitarian contribution measures presented afforded comparisons for the groups, and the sparking measures served to normalise and affirm thoughts. Of all results, all but two students registered a change, arising from exposure to different representations of the group work. These results can serve as a basis for creating future interfaces to support small group work, as well as understanding practicalities (such as the time required to reflect) and the potential need for multiple visuals, in regard to aspects deemed important for review.

\section{CONCLUSION}

With the rapid rise of data-intensive solutions aimed at enhancing human understanding of subtle and, sometimes, invisible patterns, it is critical to explore the key role of the presentation forms and the scaffolding needed to support people to make sense of their data and give meaning to their own interaction experience. This work is aimed at being one piece of much more research needed in this area. Our student-facing dashboard can serve as an exemplar of a data analytics interface that guides people with a set of scaffolded reflection activities and visualisation prompts, sitting in the context of group brainstorming. The aim is to offer people the benefit of reflection on what they did, how they did it, and what they learnt.

In our study, multiple visual OLM representations for both egalitarian participation and idea sparking led to changes in selfassessment ratings for 28 of the 30 students. The reflection after the brainstorm activity provided the opportunity to enhance each group members' understanding of key activity processes. From the results captured, both the self-reported Likert ratings and the feedback, students found utility with different visual representations: the contributions timeline allowing performance tracking; the replay activating memory; and the hands-on classification exercise highlighting the extent of group related phenomena. This study demonstrates the usefulness of showing different visualisations drawn from modelling some aspects of the group process. These were effective for gaining insights beyond those of just the final outputs arising from the brainstorm activity. There are benefits in allowing group members to watch a rapid replay of the whole activity, but in some cases, there are time restrictions that may make this not too practical. Furthermore, this work is the only study to date, which has attempted to measure the brainstorming effect of sparking, by calling on people's beliefs. 
Throughout our study, we explored how the benefits of OLMs can be applied to help groups of students reflect on critical aspects of brainstorming. Moving forward, will be the use of this in more authentic longer-term out-of-the-lab settings, utilising the lessons learned here. For future studies, it would be interesting to investigate how the reflection exercises scale with larger groups, and it would also be valuable to test with different groups of people drawn from other areas, such as a workplace, given the recent resurgence in design thinking and creative problem solving. Additionally, further work can lead to generating understanding about how the use of student-facing dashboards can play a critical role in the day-to-day facilitator-lead reflections that are quite commonly conducted as a part of classroom sessions over multiple uses.

\section{ACKNOWLEDGEMENTS}

This work was partly funded by the Smart Services CRC. This research was also supported by funding from the Faculty of Engineering \& Information Technologies, The University of Sydney, under the Faculty Research Cluster Program. The views expressed herein are those of the authors and are not necessarily those of the Faculty. The study presented was conducted with full adherence to the human ethics committee provisions (Ethics Protocol: 13275) as outlined by the University of Sydney.

\section{REFERENCES}

[1] Ammar Al-Qaraghuli, Halimah Zaman, Patrick Olivier, Ahmed Kharrufa and Azlina Ahmad. 2011. Analysing Tabletop Based Computer Supported Collaborative Learning Data through Visualization In Proceedings of Visual Informatics 2011 (IVIC 2011). Springer, 329-340.

[2] Roger Azevedo and Vincent Aleven. 2013. Metacognition and Learning Technologies: An Overview of Current Interdisciplinary Research. In International Handbook of Metacognition and Learning Technologies, Springer, 1-16.

[3] Jonali Baruah and Paul B Paulus. 2016. The Role of Time and Category Relatedness in Electronic Brainstorming. Small Group Research 47, 3, 333-342.

[4] Vincent R Brown and Paul B Paulus. 2002. Making Group Brainstorming More Effective: Recommendations from an Associative Memory Perspective. Current Directions in Psychological Science 11, 6, 208-212.

[5] Vincent Brown, Michael Tumeo, Timothy S Larey and Paul B Paulus. 1998. Modeling Cognitive Interactions During Group Brainstorming. Small Group Research 29, 4, 495-526.

[6] Stéphanie Buisine, Guillaume Besacier, Améziane Aoussat and Frédéric Vernier. 2012. How Do Interactive Tabletop Systems Influence Collaboration? Computers in Human Behavior 28, 1, 49-59. http://dx.doi.org/http://dx.doi.org/10.1016/j.chb.2011.08.010

[7] Susan Bull and Mark Britland. Year. Group Interaction Prompted by a Simple Assessed Open Learner Model That Can Be Optionally Released to Peers. In Proceedings of Proceedings of Workshop on Personalisation in E-Learning Environments at Individual and Group Level (PING), User Modeling.

[8] Susan Bull, Blandine Ginon, Judy Kay and Michael Kickmeier-Rust. 2016. Lal Workshop: Learning Analytics for Learners. In Proceedings of International Conference on Learning Analytics and Knowledge. 496-497.

[9] Susan Bull and Judy Kay. 2007. Student Models That Invite the Learner In: The Smili Open Learner Modelling Framework. International Journal of Artificial Intelligence in Education 17, 2, 89-120.

[10] Susan Bull and Judy Kay. 2008. Metacognition and Open Learner Models. In Proceedings of 3rd Workshop on Meta-Cognition and Self-Regulated Learning in Educational Technologies - Conference on Intelligent Tutoring Systems (ITS'08). Montreal, Canada.

[11] Susan Bull and Judy Kay. 2010. Open Learner Models. In Advances in Intelligent Tutoring Systems, Springer, 301-322.

[12] Susan Bull and Judy Kay. 2013. Open Learner Models as Drivers for Metacognitive Processes. In International Handbook of Metacognition and Learning Technologies, Roger Azevedo and Vincent Aleven Eds. Springer New York, New York, NY, 349-365. http://dx.doi.org/10.1007/978-1-4419-5546$\underline{3 \_} 23$
[13] Susan. Bull and R. Vatrapu. 2011. Supporting Collaborative Interaction with Open Learner Models: Existing Approaches and Open Questions. In Proceedings of 9 th International Conference on Comupter Supported Collaborative Learning 2011 (CSCL 2011). Hong Kong, China, 761-765.

[14] A. Clayphan, A. Collins, C. Ackad, B. Kummerfeld and J. Kay. 2011. Firestorm: A Brainstorming Application for Collaborative Group Work at Tabletops. In Proceedings of International Conference on Interactive Tabletops and Surfaces 2011 (ITS 2011). ACM, Kobe, Japan, 162-171.

[15] Andrew Clayphan, Judy Kay and Armin Weinberger. 2014. Scriptstorm: Scripting to Enhance Tabletop Brainstorming. Personal and Ubiquitous Computing 18, 6, 1433-1453.

[16] Andrew Clayphan, Roberto Martinez-Maldonado and Judy Kay. Year. Designing Olms for Reflection About Group Brainstorming at Interactive Tabletops. In Proceedings of AIED 2013 Workshops Proceedings. Citeseer, 37.

[17] Andrew Clayphan, Roberto Martinez-Maldonado and Judy Kay. 2013. Open Learner Models to Support Reflection on Brainstorming at Interactive Tabletops. In Proceedings of International Conference on Artificial Intelligence in Education 2013 (AIED 2013). 683-686.

[18] Andrew Clayphan, Roberto Martinez-Maldonado, Martin Tomitsch, Susan Atkinson and Judy Kay. 2016. An in-the-Wild Study of Learning to Brainstorm: Comparing Cards, Tabletops and Wall Displays in the Classroom. Interacting with Computers 28, 6, 788-810. http://dx.doi.org/10.1093/iwc/iww001

[19] J Dewey. 1933. How We Think: A Restatement of the Relation of Reflective Thinking to the Educative Process. Boston: Dc Heath and Company. An Analysis of The Content of The Curriculum for The Primary Phase in Turkey 49.

[20] Joan Morris DiMicco and Walter Bender. Year. Group Reactions to Visual Feedback Tools. In Proceedings of International Conference on Persuasive Technology. Springer, 132-143.

[21] Son Do-Lenh. 2012. Supporting Reflection and Classroom Orchestration with Tangible Tabletops. PhD thesis. École Polytechnique Fédérale de Lausanne,

[22] Karen Leggett Dugosh and Paul B Paulus. 2005. Cognitive and Social Comparison Processes in Brainstorming. Journal of experimental social psychology 41, 3, 313-320.

[23] Karen Leggett Dugosh, Paul B Paulus, Evelyn J Roland and Huei-Chuan Yang. 2000. Cognitive Stimulation in Brainstorming. Journal of Personality and Social Psychology 79, 5, 722 .

[24] T Erickson, DN Smith, WA Kellogg, M Laff, JT Richards and E Bradner. 1999. Socially Translucent Systems: Social Proxies, Persistent Conversation, and the Design of "Babble". In Proceedings of CHI 1999 Conference on Human Factors in Computer Systems. ACM, Pittsburgh, Pennsylvania, USA, 72-79.

[25] Otmar Hilliges, Lucia Terrenghi, Sebastian Boring, David Kim, Hendrik Richter and Andreas Butz. 2007. Designing for Collaborative Creative Problem Solving. In Proceedings of Proceedings of the 6th ACM SIGCHI conference on Creativity \& Cognition. ACM, Washington, DC, USA, 137-146.

[26] P. Isenberg, N. Elmqvist, J. Scholtz, D. Cernea, Ma Kwan-Liu and H. Hagen. 2011. Collaborative Visualization: Definition, Challenges, and Research Agenda. Information Visualization 10, 4, 310-326. http://dx.doi.org/10.1177/1473871611412817

[27] Amandine Afonso Jaco, Stéphanie Buisine, Jessy Barré, Améziane Aoussat and Frédéric Vernier. 2014. Trains of Thought on the Tabletop: Visualizing Association of Ideas Improves Creativity. Personal and Ubiquitous Computing $18,5,1159-1167$

[28] Judy Kay and Susan Bull. 2015. New Opportunities with Open Learner Models and Visual Learning Analytics. In Proceedings of International Conference on Artificial Intelligence in Education. Madrid, Spain 666-669.

[29] A.S. Kharrufa, P. Olivier and D. Leat. 2010. Learning through Reflection at the Tabletop: A Case Study with Digital Mysteries. In Proceedings of World Conference on Educational Multimedia, Hypermedia and Telecommunications 65-674.

[30] Cole Nussbaumer Knaflic. 2015. Storytelling with Data: A Data Visualization Guide for Business Professionals. John Wiley \& Sons,

[31] Nicholas W Kohn, Paul B Paulus and YunHee Choi. 2011. Building on the Ideas of Others: An Examination of the Idea Combination Process. Journal of Experimental Social Psychology 47, 3, 554-561.

[32] Yanjin Long and Vincent Aleven. Year. Students' Understanding of Their Student Model. In Proceedings of Artificial Intelligence in Education. Springer, 179-186.

[33] Andrew Mabbott and Susan Bull. Year. Alternative Views on Knowledge: Presentation of Open Learner Models. In Proceedings of Intelligent Tutoring Systems. Springer, 689-698.

[34] R. Martinez-Maldonado, A. Clayphan, K. Yacef and J. Kay. 2015. Mtfeedback: Providing Notifications to Enhance Teacher Awareness of Small Group Work in the Classroom. IEEE Transactions on Learning Technologies 8, 2, 187-200. http://dx.doi.org/10.1109/TLT.2014.2365027

[35] Roberto Martinez-Maldonado, Judy Kay, Kalina Yacef, Marie-Theresa Edbauer and Yannis Dimitriadis. 2012. Orchestrating a Multi-Tabletop Classroom: From Activity Design to Enactment and Reflection. In Proceedings of International 
Conference on Interactive Tabletops and Surfaces 2012. ACM, Cambridge, USA, $119-128$

[36] Roberto Martinez-Maldonado, James Wallace, Judy Kay and Kalina Yacef. 2011. Modelling and Identifying Collaborative Situations in a Collocated MultiDisplay Groupware Setting. In Proceedings of International Conference on Artificial Intelligence in Education 2011 (AIED 2011). ACM, 196-204.

[37] Roberto Martinez Maldonado, Judy Kay, Kalina Yacef and Beat Schwendimann. Year. An Interactive Teacher's Dashboard for Monitoring Groups in a Multi-Tabletop Learning Environment. In Proceedings of Intelligent Tutoring Systems. Springer Berlin Heidelberg, 482-492. http://dx.doi.org/10.1007/978-3-642-30950-2_62

[38] Jack Mezirow. 1991. Transformative Dimensions of Adult Learning. ERIC

[39] Bernard A Nijstad and Wolfgang Stroebe. 2006. How the Group Affects the Mind: A Cognitive Model of Idea Generation in Groups. Personality and social psychology review 10, 3, 186-213.

[40] A.F. Osborn. 1953. Applied Imagination: Principles and Procedures of Creative Problem Solving. Charles Scribener's Sons, New York.

[41] Paul B Paulus and Vincent R Brown. 2007. Toward More Creative and Innovative Group Idea Generation: A Cognitive- Social- Motivational Perspective of Brainstorming. Social and Personality Psychology Compass 1, 1, 248-265.

[42] Paul B Paulus, Daniel S Levine, Vincent Brown, Ali A Minai and Simona Doboli. 2010. Modeling Ideational Creativity in Groups: Connecting Cognitive, Neural, and Computational Approaches. Small Group Research 41, 6, 688-724.

[43] Paul B Paulus and Bernard A Nijstad. 2003. Group Creativity: Innovation through Collaboration. Oxford University Press,
[44] Paul B Paulus, Vicky L Putman, Karen Leggett Dugosh, Mary T Dzindolet and Hamit Coskun. 2002. Social and Cognitive Influences in Group Brainstorming: Predicting Production Gains and Losses. European review of social psychology Predicting Pro $1,299-325$

[45] Paul B Paulus and Huei-Chuan Yang. 2000. Idea Generation in Groups: A Basis for Creativity in Organizations. Organizational behavior and human decision processes $82,1,76-87$.

[46] Jeroen G Raaijmakers and Richard M Shiffrin. 1981. Search of Associative Memory. Psychological review 88, 2, 93.

[47] Lara Schmitt, Stéphanie Buisine, Jonathan Chaboissier, Améziane Aoussat and Frédéric Vernier. 2012. Dynamic Tabletop Interfaces for Increasing Creativity. Computers in Human Behavior 28, 5, 1892-1901.

[48] Donald A Schön. 1983. The Reflective Practitioner: How Professionals Think in Action. Basic books,

[49] Sarah Tausch, Stephanie Ta and Heinrich Hussmann. 2016. A Comparison of Cooperative and Competitive Visualizations for Co-Located Collaboration. In Proceedings of Proceedings of the 2016 CHI Conference on Human Factors in Computing Systems. ACM, Santa Clara, California, USA, 5034-5039.

[50] Hao-Chuan Wang, Tsai-Yen Li, Carolyn P Rosé, Chun-Chieh Huang and ChunYen Chang. Year. Vibrant: A Brainstorming Agent for Computer Supported Creative Problem Solving. In Proceedings of Intelligent Tutoring Systems. Springer, 787-789.

[51] Robert W Weisberg. 1999. Creativity and Knowledge: A Challenge to Theories Handbook of creativity. 